\title{
Comparison of Clinical Characteristics and Outcomes in Patients with STEMI in Croatia and Kosovo
}

\author{
Rreze Koshi $^{1^{*}}$, Maja Strozzi ${ }^{2}$, Davor Milicic ${ }^{3}$ and Shpend Elezi ${ }^{4}$ \\ ${ }^{I}$ Department of Cardiology, Regional Hospital Isa Grezda, Gjakova, Kosovo \\ ${ }^{2}$ Department of Cardiology, Zagreb University Hospital Center, Zagreb, Croatia \\ ${ }^{3}$ Department for Cardiovascular Diseases, University Clinical Center of Kosovo, Prishtina, Kosovo \\ ${ }^{4}$ Department for Internal Medicine, University of Prishtina, Kosovo
}

Correspondence should be addressed to Rreze Koshi, rreze.koshi@gmail.com

Received Date: June 30, 2020; Accepted Date: July 13, 2020; Published Date: July 20, 2020

\begin{abstract}
$\underline{\text { ABSTRACT }}$
INTRODUCTION

The aim of our study was to compare clinical characteristics, risk factors, treatment modalities and medium-term outcomes in STEMI patients in two different countries, Croatia and Kosovo.
\end{abstract}

\section{MATERIAL AND METHODS}

The study included 77 consecutive patients from two regional hospitals, included in the STEMI network of University Clinical Center Zagreb, Croatia (group 1), and 75 STEMI consecutive patients treated in regional Hospital Gjakova, Kosovo (group 2). Standard laboratory tests were performed in both study groups. Patients were treated by the standard protocol of the country and hospital where they were treated. Immediate clinical outcomes and 6 months follow up results regarding MACE were compared.

\section{RESULTS}

There was no significant difference between groups in basic clinical characteristic except for diabetes mellitus, $\left(\mathrm{x}^{2}=6.96, \mathrm{P}=\right.$ $0.008, \mathrm{P}<0.01$ ) and glycaemia control ( $\mathrm{U}^{\prime}=1530, \mathrm{P}=0.013$ ). No significant difference between two groups in laboratory findings. There was a difference in percent of patients treated with primary PCI, 71 in gr 1 and 55 in group 2, reaching statistical significance $\left(\mathrm{x}^{2}\right.$ test $\left.8,261, \mathrm{p}=0.0045\right)$. In patients treated with PCI, there was no difference in "door to balloon time" (DTB) between groups. There was a remarkable difference in proportion of drug eluting stent (DES) implantation (3.5\% in group 1, $29.1 \%$ in group 2 ( $\mathrm{x}^{2}$ test $13.5, \mathrm{P}=0.0002$ ). The difference partly derives from the relatively large number of balloon interventions in group 2 . There was 2 deaths in group 1 in early hospital period, both during interventional procedure, one of the patients was in cardiogenic shock. There was no early mortality in group 2. In 6 month clinical follow up there were 3 deaths in group 2, all in patient with no revascularization, and no death in group 1.

\section{CONCLUSION}

We compared the clinical characteristics, treatment strategies and outcomes, in STEMI patients from two countries. After initial mortality, patients from Croatia had a better mid-term survival after recovery from their AMI compared to patients from Kosovo. Use of revascularization procedures was beneficial, but it was less often performed in Kosovo.

Citation: Rreze Koshi, Comparison of Clinical Characteristics and Outcomes in Patients with STEMI in Croatia and Kosovo. J Heart 1(1): 24-28. 


\section{KEYWORDS}

STEMI; Primary PCI; Cardiovascular risk

\section{INTRODUCTION}

Acute myocardial infarction (AMI) remains a major global cause of death and disability. Overall mortality in AMI has reduced in the last several decades as a consequence of advancement in treatment, especially after introduction of primary PCI in STEMI.

The aim of this study was to compare clinical characteristics, risk factors, treatment modalities and prognosis in STEMI patients in two different countries: Croatia and Kosovo. The patients were analyzed for several clinical and treatment characteristics in the acute phase, and at follow-up after 6 months. Similarly, the rate of patients reperfused in a timely manner also increased, with a significant reduction in door to needle and door to artery times [1]. Factors contributing to the sex difference of in-hospital mortality after acute myocardial infarction are still unknown [2]. No differences were observed between men and women with respect to age, history of diabetes, hypertension, and lipid disorders in patients with a confirmed AMI. Previous MI, smoking, and history of CHF were more frequent in men [3].

\section{MATERIAL AND METHODS}

The study included 152 consecutive patients with STEMI, 77 patients treated in University Clinical Center Zagreb, Croatia (group 1), and 75 patients treated in Hospital Gjakova, Kosovo. Study was performed form 1 June 2014, until patients in both groups are included. All patients from University Clinical Center were first diagnosed with acute infarction in two regional hospitals (General hospital Varazdin and Bjelovar), transported for primary coronary intervention (Croatian STEMI network). These patients from regional hospitals were better matched with patients from a regional hospital in Kosovo. Patients from regional hospital Isa Grezda,
Gjakova were treated with primary PCI in University hospital in Pristina, or treated conservatively.

Demographic and clinical data were obtained, (table 1) laboratory test were performed in both study groups (table 2), treatment modalities, compared PCI with conservative therapy (table 3), as well as clinical outcomes 6 month after STEMI, regarding major adverse events (MACE): death, re-infarction, stroke or re-intervention (table 4).

\section{Coronary angiography}

It was performed in all Croatian patients and in $73.3 \%$ of patients from Kosovo. The perfusion status of the infarct related artery was assessed and PCI with stenting was the first choice therapy, where which was possible. Part of Kosovo patient did not receive any reperfusion therapy because lack of interventional facilities and network organization, or problems with procedural costs. Treatment modalities are summarized in table 3 .

\begin{tabular}{|c|c|c|c|}
\hline & Zagreb (Group 1) & Kosovo (Group 2) & $\mathrm{p}$ value \\
\hline Gender (male/female) & $54 / 23(70.13 / 29.87 \%) \mathrm{p}>0.872$ & $63 / 12(84 / 16 \%) \mathrm{p}>0.898$ & \\
\hline Age & $60.1(\mathrm{SD} \pm 12.5)$ & $61.4(\mathrm{SD} \pm 12.5)$ & $\mathrm{p}=0.872$ \\
\hline Diabetes mellitus & $26(33.77 \%)$ & $45(60 \%)$ & $\mathrm{p}=0.008$ \\
\hline Hypertension & $47(61.04 \%)$ & $52(69.33 \%)$ & $\mathrm{p}=0.398$ \\
\hline Hyperlipidemia & $40(51.95 \%)$ & $44(58.67 \%)$ & $\mathrm{p}=0.557$ \\
\hline Smoking & $39(50.65 \%)$ & $45(60 \%)$ & $\mathrm{p}=0.551$ \\
\hline Positive family history & $36(46.75 \%)$ & $33(44 \%)$ & $\mathrm{p}=0.8$ \\
\hline
\end{tabular}

Table 1: Baseline characteristics.

\begin{tabular}{|c|c|c|c|}
\hline & Zagreb (Group 1) & Kosovo (Group 2) & p value \\
\hline $\mathrm{CK} \mathrm{mmol} / \mathrm{L}$ & $1691.4(\mathrm{SD} \pm 1687.8)$ & $500.7(\mathrm{SD} \pm 615.3)$ & $\mathrm{U}^{\prime}=1885.0, \mathrm{P}<0.0001$ \\
\hline $\mathrm{LDH} \mathrm{mmol} / \mathrm{L}$ & $433.7(\mathrm{SD} \pm 269.2)$ & $617.3(\mathrm{SD} \pm 495.0)$ & $\mathrm{U}^{\prime}=1237.0, \mathrm{P}=0.213$ \\
\hline Troponin & $2.77(\mathrm{SD} \pm 2.70)$ & $2.43(\mathrm{SD} \pm 3.12)$ & $\mathrm{U}^{\prime}=139.0, \mathrm{P}=0.5258$ \\
\hline CRP positive (\% patients) & 73.7 & 67.3 & $\mathrm{X}^{2}=0.288, \mathrm{P}=0.591$ \\
\hline Cholesterol mmol/L & $5.06(\mathrm{SD} \pm 1.23)$ & $5.40(\mathrm{SD} \pm 1.78)$ & $\mathrm{T}=0.738, \mathrm{P}=0.464$ \\
\hline Triglycerides mmol/L & $1.56(\mathrm{SD} \pm 0.74)$ & $1.64(\mathrm{SD} \pm 0.67)$ & $\mathrm{U}^{\prime}=335.5, \mathrm{P}=0.407$ \\
\hline Glycemia mmol/L & $7.3(\mathrm{SD} \pm 2.8)$ & $10.4(\mathrm{SD} \pm 5.6)$ & $\mathrm{U}^{\prime}=1530.5, \mathrm{P}=0.0013$ \\
\hline Uric acid mmol/L & $321.1(\mathrm{SD} \pm 91.5)$ & $310.1(\mathrm{SD} \pm 121.8)$ & $\mathrm{U}^{\prime}=225.5, \mathrm{P}=0.489$ \\
\hline
\end{tabular}

Table 2: Laboratory findings. 


\begin{tabular}{|c|c|c|c|}
\hline & Zagreb (Group 1) & Kosovo (Group 2) & p value \\
\hline PCI traated patients & 74 & 55 & \\
\hline DBT (hours) & $5.2(\mathrm{SD} \pm 7.2)$ & $6.6(\mathrm{SD} \pm 8.6)$ & $\mathrm{U}^{\prime}=1009.5, \mathrm{P}=0.809$ \\
\hline DES $/$ BMS (\%) & $60(84.4)$ & $14(25.5)$ & $\mathrm{X}^{2}=13.5, \mathrm{P}=0.0002$ \\
& $2(2.8)$ & $16(29)$ & \\
& $9(12.8)$ & $25(45.5)$ & \\
& & & \\
Culprit vessel N/\% & $27(38)$ & $23(41.8)$ & \\
LAD & $28(39,5)$ & $8(14.5)$ & \\
CX & $12(17)$ & $16(29.1)$ & \\
Other & $4(5.5)$ & $8(14.5)$ & \\
\hline
\end{tabular}

Table 3: Interventional procedure data.

\begin{tabular}{|c|c|c|c|}
\hline & Zagreb (Group 1) & Kosovo (Group 2) & $\mathrm{p}$ value \\
\hline MACE & 13 & 12 & $\mathrm{p}=0.9447$ \\
\hline Death (early and 6 months FU) & 2 & 3 & $\mathrm{p}=0.9766$ \\
\hline Re-intervention & 8 & 4 & $\mathrm{p}=0.39$ \\
\hline CABG & 3 & 4 & $\mathrm{p}=0.9726$ \\
\hline Stroke & 0 & 1 & $\mathrm{p}=0.9897$ \\
\hline
\end{tabular}

Table 4: 6 months outcome (immediate and follow up results).

\section{Statistical analysis}

Categorical variables were compared using the $\chi^{2}$ test. A probability value of $p<0.05$ was considered to indicate a statistically significant difference. Continuous variables were compared using the unpaired $t$ test and Main Whitney U test.

\section{RESULTS}

Regarding gender in both groups there was a greater share of male patients, with higher prevalence of male patients in Kosovo group. Patients in this group were older, but without reaching statistical difference. $(\mathrm{T}=0.738, \mathrm{P}=$ 0.464).There was a significant difference in diabetes mellitus in favor of Kosovo group $\left(\mathrm{x}^{2}=6.96, \mathrm{P}=0.008, \mathrm{P}\right.$ $<0.01)$.There was no difference in other risk factors between the groups (smoking, hypertension and family history). All laboratory values where similar in both groups (troponin, CK, LDH, CRP, uric acid, cholesterol, triglyceride), where similar in both groups, with exception of the average value of the glycaemia $\left(\mathrm{U}^{\prime}=1530, \mathrm{P}=\right.$ $0.013)$.

Door to balloon time was not different, (U' $=1009.5, \mathrm{P}=$ 0.809), but in Kosovo group there was a large dispersion of data (2-48 hours). In Zagreb group in all patients an invasive coronary study was performed, in 6 patients no intervention was indicated. The reason to defer the intervention was: no coronary lesion in three patients, one of them with myocarditis and one with severe aortic stenosis, diffuse disease with no culprit lesion in two patient, and in one patient emergency surgery was performed. In majority of patients a primary PCI with stent implantation was performed. In Kosovo group some patients remain not re-perfused. In patients with primary PCI, there was a difference between groups according the type of stents implanted. DES was implanted in $2.8 \%$ of patients and $84.2 \%$. BMS in patients from the UCC Zagreb, comparing with patients from Kosovo, where $29.1 \%$ implanted stents where drug eluting, and $25.5 \%$ BMS. In group 2 there was patients with just balloon angioplasty done. This reached significant statistical difference $\left(\mathrm{x}^{2}\right.$ test $\left.=13.5, \mathrm{P}=0.0002, \mathrm{P}<0.01\right)$.

The clinical outcome were compared after 6-month follow up (FU) (table 4). In this period there was 4 deaths in group 2, and no additional death in group 2. In both group there was need for surgical revascularization, in 4 patients from Kosovo, respectively 3 patients from Zagreb. Re- intervention was performed in 8 patients from group 1during FU. In majority of group 1 patients (6) it was a non-culprit lesion elective intervention. In the same period in 4 patients in group 2 re-intervention was performed, all in culprit lesion (3 stent thrombosis cases). One patient from group 2 suffered from stroke. So, cumulative MACE was not significantly different between two groups, mostly due to higher percentage of PCI treated patients in Zagreb. As mentioned above these two type odd PCI cannot be compared. 


\section{DISCUSSION}

Cardiovascular disease (CVD) is an important cause of death among both men and women. In women, CVD develops 7 years to 10 years later than in men, potentially because of a protective effect of estrogens [4]. In comparison of demographic characteristics we found that Kosovo group was older and has fewer females. We cannot explain the reason for older age (possible lower atherosclerotic burden). Gender difference can be explained with the cultural background (may be women did not searched for help or infarction was not recognized by medical stuff). There was no difference in risk factors except for diabetes, what can be result of lower quality of prevention, and the worse glycaemia control can be connected with unsatisfactory health insurance system. The difference in percent of reperfusion can be for the same reason. Limited data exist on contemporary sexrelated differences in long-term outcomes of coronary patients receiving drug-eluting stents [5]. Croatia has a good organized network for primary PCI, in Kosovo that depend on working hours and individual effort. This is a reason for differences in door to balloon time, but it has not reached statistical difference.

The choice of the stent was to operator discretion in both groups (DES or BMS) using the standard techniques following antithrombotic and antiplatelet therapy. It is known that DES has remarkably reduced the incidence of the restenosis compared with BMS in randomized trials.
There was a difference in use of DES, in favor of Kosovo. At that time there was a budget restriction in Zagreb, so majority of patient get a BMS. In Kosovo, in patients treated with primary PCI in University Clinical center, more DES was implanted. Despite well-established guidelines for the management of STEMI, there are still differences with regard to the epidemiology, diagnostics and treatment of patients, leading to diverging morbidity and mortality rates across the globe. Not all reasons for these differences are well understood [6].

\section{CONCLUSION}

There is a significant difference in clinical characteristic of STEMI patients in two different countries that use to be in the same health system 25 years ago. The differences are: older population, less presentation of women in Kosovo. There was not difference in risk factor profile, except for diabetes (incidence and glycaemia control). There were less re-perfused patients in Kosovo, some difference in DTB time. Some of these differences are due to different health system, and organization of STEMI care. Difference in mentality and social status can also have some influence on STEMI treatment and outcomes. Different stent use (more DES in Kosovo, more intervention with BMS in Zagreb) can also illustrate these differences. The socioeconomic differences in the use of revascularization procedures could not be explained by differences in co-morbidity or type of hospital at first admission [7].

\section{REFERENCES}

1. Schiele F, Hochadel M, Tubaro M, et al. (2010) Reperfusion strategy in Europe: Temporal trends in performance measures for reperfusion therapy in ST-elevation myocardial infarction. European Heart Journal 31(21): 2614-2624.

2. Shiraki T, Saito D (2011) Sex difference of in-hospital mortality in patients with acute myocardial infarction. Acta Medica Okayama 65(5): 307-314.

3. Romero T, Velez P, Glaser D, et al. (2013) Do gender and race/ethnicity influence acute myocardial infarction quality of care in a hospital with a large Hispanic patient and provider representation?. Cardiology Research and Practice. 
4. Claassen M, Sybrandy KC, Appelman YE, et al. (2012) Gender gap in acute coronary heart disease: Myth or reality?. World Journal of Cardiology 4(2): 36.

5. Shammas NW (2012) Outcomes in contemporary coronary interventions with drug-eluting stents for acute myocardial infarction: The gender role. Journal of Invasive Cardiology 24(3): 108-109.

6. Kämpfer J, Yagensky A, Zdrojewski T, et al. (2017) Long-term outcomes after acute myocardial infarction in countries with different socioeconomic environments: An international prospective cohort study. BMJ Open 7(8): $\mathrm{e} 012715$.

7. Rosvall M, Chaix B, Lynch J, et al. (2008) The association between socioeconomic position, use of revascularization procedures and five-year survival after recovery from acute myocardial infarction. BMC Public Health 8(1): 44. 